\title{
The sensitivity to inter-subject variability of the bridging vein entry angles for prediction of acute subdural hematoma.
}

\author{
Markos KAPELIOTIS ${ }^{a, *}$, Gracia Umuhire MUSIGAZI ${ }^{\mathrm{b}}$, Nele FAMAEY ${ }^{\mathrm{a}}$, Bart DEPREITERE ${ }^{\mathrm{b}}$, \\ Svein KLEIVEN ${ }^{c} \&$ Jos VANDER SLOTEN ${ }^{a}$ \\ ${ }^{a}$ Biomechanics Section, KU Leuven, Belgium \\ ${ }^{b}$ Department of Neurosurgery, University Hospital Gasthuisberg, KU Leuven, Belgium \\ ${ }^{c}$ Neuronic engineering, School of Technology and Health, KTH Royal Institute of Technology, Sweden
}

\begin{abstract}
Acute subdural hematoma (ASDH) is one of the most frequent traumatic brain injuries (TBIs) with high mortality rate. Bridging vein (BV) ruptures is a major cause of ASDH. The KTH finite element head model includes bridging veins to predict acute subdural hematoma due to BV rupture. In this model, BVs were positioned according to Oka et al. 10 . The aim of the current study is to investigate whether the location and entry angles of these BVs could be modelled using data from a greater statistical sample, and whe impact of this improvement would be on the model's predictive capability of BV rupture.

From the CT angiogram data of 78 patients, the relative position of the bridging veins and their entry angles along the superior sagittal sinus was determined. The bridging veins were repositioned in the model accordingly. The performance of the model, w.r.t. BV rupture prediction potential was tested on simulations of full body cadaver head impact experiments. The experiments were simulated on the original version of the model and on three other versions which had updated BV positions according to mean, maximum and minimum entry angles.

Even though the successful prediction rate between the models stayed the same, the location of the rupture site significantly improved for the model with the mean entry angles. Moreover, the models with maximum and minimum entry angles give an insight of how BV biovariability can influence ASDH.

In order to further improve the successful prediction rate, more biofidelic data are needed both with respect to bridging vein material properties and geometry. Furthermore, more experimental data are needed in order to investigate the behaviour of FE head models in depth.
\end{abstract}

Keywords: head impact, acute subdural hematoma, bridging vein rupture, CT angiogram, bridging vein position, finite element head model

\section{Introduction}

Acute subdural hematoma (ASDH) is one of the most frequent traumatic brain injuries (TBIs) with mortality rates ranging from $30 \%$ to $90 \%$. Next to head contusion and laceration of cerebral veins and arteries, rupture of a bridging vein (BV) is a major cause of ASDH 3 . 8 .

Finite element (FE) models of the human head are used to study head injuries such as ASDH. Most of the FE head models include a mechanical representation of bridging veins (BVs). The SIMon model ${ }^{11}$ has BVs modelled as cable discrete beams with a Youngs modulus of $0.275 \mathrm{MPa}$. The

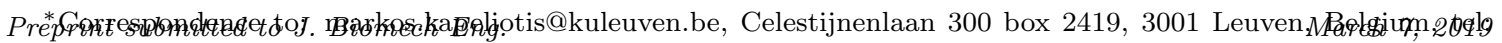
+3216376068 
KTH FE head model $\left[\right.$ has discrete beam elements with a stiffness of $1.9 \mathrm{~N}$, while the UCDBTM ${ }^{5}$ and the WSUBIM ${ }^{12}$ also have similar BV representations. The only study investigating the reliability of head models on BV ruptures has been the one of Cui et al. 1], where data from whole body cadaver experiments were used to assess the predictive capability of the KTH head model by testing different reported material properties.

Another factor that can affect the outcome, and has not been investigated so far, is the BV positioning within the models. All head models to the authors knowledge have used the findings 45 from Oka 10 to position bridging veins. Musigazi et al. 9 performed a study where the venous phase of CT angiograms of the heads of 78 patients were retrieved from the hospital database of the University hospital in Leuven. The relative position of the BVs and their entry angles along the superior sagittal sinus (SSS) was determined, referring to conventional anatomical landmarks.

This study evaluates the representation of BVs in the KTH head model in three cases: i.e. when

so the mean entry angles of BVs are used, when the minimum observed entry angles are used, and when the maximum observed entry angles are used. In every case the BVs are distributed throughout the cortex using the mean number of BVs per cortical region as was reported by Musigazi et al. 9] . These models are then compared to the original head model to assess if positioning can improve its predictive capability.

\section{Material and Methods}

Musigazi et al. 9 performed a study on BV anatomy based on CT angiogram data from 78 patients from the university hospital of Leuven. Using the nasion and inion as anamical landmarks the cortex was split into ten segments, each being a cortical region of $18^{\circ}$. The BVs were categorized per segment and their entry angle to the SSS was measured (See Fig: 1). It was ${ }_{60}$ reported that BVs were distributed along the SSS in segments 1 to 8. Most BVs were located in segment $5(30 \%)$ and in segments 4 and $6(20 \%)$. There were 0-3 BVs, on average, per segment and side. The median angle of entrance with respect to the SSS $\left(180^{\circ}=\right.$ frontal, $0^{\circ}=$ occipital direction $)$ varied between $63-86^{\circ}$ (frontal) and $44-76^{\circ}$ (parietal).

A detailed description of the KTH FE head model can be found in 6 . The original model has the BVs positioned according to the findings reported by Oka 10 . By using the findings reported by Musigazi et al. 9 the location and entry angles of the BVs to the SSS were changed. In order to achieve these angles, the beam elements are connected to free nodes placed on the cortical surface. These nodes are connected to nodes of the cortical surface by constrained interpolation. With this constraint type, the motion of a single dependent node is interpolated from the motion of a set of independent nodes 7 (See Fig: 2). Constrained interpolation was also used on the original BV configuration to investigate if this specific constraint could affect the outcome of the simulations.

Three different versions of the model were produced using the results from this study, one matching the reported mean angles, one with the maximum observed angles and one with the minimum observed angles. Mainly, the comparison between the original and the mean angles model is of interest due to the likelihood of the BVs being in these positions. The extreme angles models are used to investigate the effect of biovariability in the way BVs are positioned on the cortex.

The commercial FE solver LS-DYNA was used. Boundary prescribed motion was used to apply the same translational and rotational accelerations. The Youngs modulus and cross sectional area reported by Monea et al. (2014) 4 were used to model the BVs. As rupture criterion the 


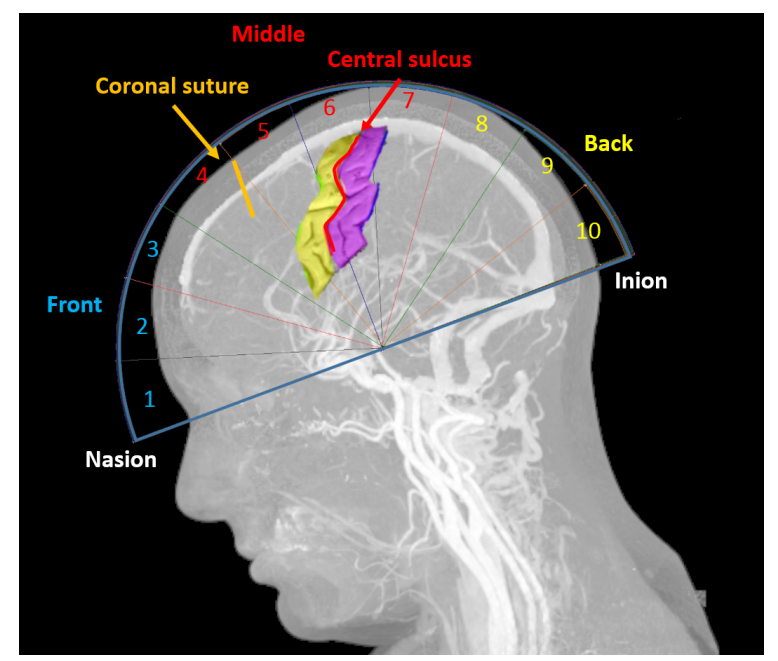

Figure 1: From CT angiograms the relative position and entry angle to the SSS was visible. The bridging veins located were categorized in 10 cortical segments.

ultimate strain reported by the same study was used $(29.82 \pm 113.26 \%)$. The strain calculated was the engineering strain of the elements. The maximum strain was considered to be the maximum positive value of all BVs over the duration of the entire simulation.
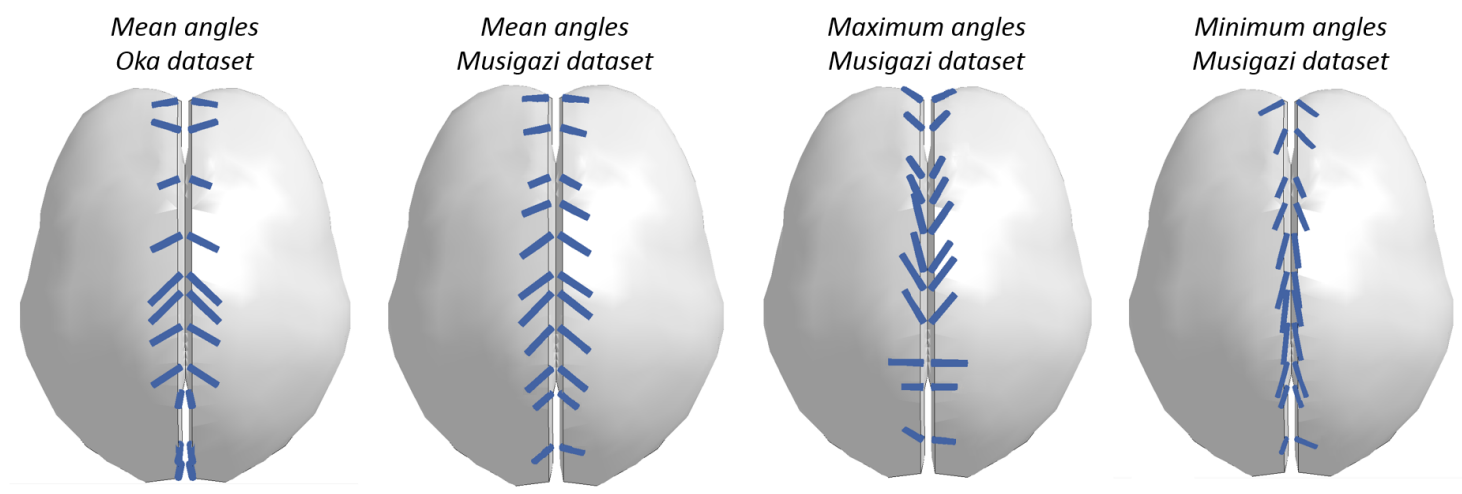

Figure 2: KTH head model with all BV configurations, from left to right: Mean angles Oka(original configuration), Mean angles Musigazi, Maximum angles Musigazi, Minimum angles Musigazi

To assess these adaptations the cadaver experiments performed by Depreitere et al. $\underline{3}$ were simulated by all the models and then their performance was compared to the original. In these experiments whole body human cadavers were placed in an upright sitting position and were impacted on the back of the head by a pendulum. The accelerations were measured using three uniaxial accelerometers which were mounted on the left side of the head (See Fig]3).This study resulted in bridging vein ruptures with known translational and rotational accelerations, which were applied on the models in order to estimate their predictive capability on bridging vein ruptures. 

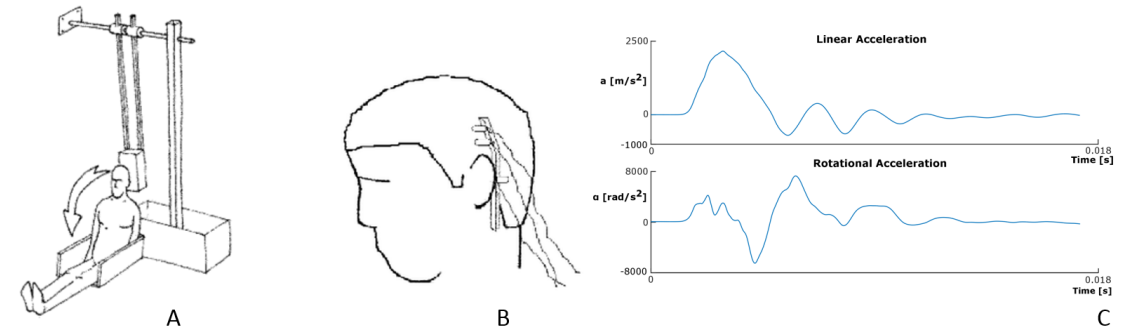

Figure 3: Cadaver impact experiments sketch from 3 . (A) Cadaver and pendulum set-up. (B) The three uni-axial accelerometers system fixed onto the cadaver head (C) Raw linear and rotational acceleration pulses from the cadaver impact experiments

The rotational acceleration was measured as the sum of the upper and the lower accelerometer divided by their relative distance, the acceleration measurements of the middle accelerometer were used to estimate the tangential acceleration of the heads centre of mass. After each blow the cadavers were checked for BV ruptures by means of fluoroscopy. The BV layout of each of the cadavers was not captured or recorded in any way.

On 10 cadavers 18 original impacts were performed. When the first impact did not produce a rupture the cadaver was impacted again. These second impacts are not considered in this study because the applied damage from the first impact cannot be accounted for.All rupture sites are located in the pre and post Rolandic region, as reported by Depreitere et. al. 3] This area corresponds to the yellow and purple area in Figure 1 and should include segments 4,5,6 and 7. The cases that were simulated are shown in table 1

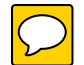

Table 1: The impact parameters of the 8 cadaver impact experiments used for this study

\begin{tabular}{|c|c|c|c|c|c|c|}
\hline $\begin{array}{l}\text { Specimen } \\
\text { no. }\end{array}$ & $\begin{array}{l}\text { Age } \\
\text { y.o. }\end{array}$ & Sex Gender & $\begin{array}{l}\text { Peak rotational } \\
\text { velocity }(\mathrm{rad} / \mathrm{s})\end{array}$ & $\begin{array}{c}\text { Peak rotational } \\
\text { acceleration }\left(\operatorname{rad}^{2} / \mathrm{s}\right)\end{array}$ & $\begin{array}{c}\text { Pulse } \\
\text { duration }(\mathrm{ms})\end{array}$ & Rupture \\
\hline 01-3_1 & 80 & $\mathrm{~F}$ & 48.21 & 15156 & 12.0 & - \\
\hline 21-3_1 & 93 & $\mathrm{~F}$ & 17.89 & 2454 & 10.2 & - \\
\hline 22-3_1 & 70 & $\mathrm{~F}$ & 17.32 & 3044 & 5.5 & - \\
\hline 25-2_1 & 65 & M & 14.18 & 7312 & 4.5 & - \\
\hline 28-2_1 & 93 & $\mathrm{~F}$ & 24.88 & 12867 & 15.4 & + (right) \\
\hline 29-3_1 & 80 & M & 57.37 & 19572 & 7.7 & + (right) \\
\hline 30-2_1 & 86 & $\mathrm{~F}$ & 19.32 & 4950 & 10.4 & - \\
\hline 32-2_1 & 55 & M & 19.19 & 6244 & 11.9 & - \\
\hline
\end{tabular}

The aim is to determine how many successful predictions of either rupture or non-rupture the adapted models can yield, with a maximum of 8 . In Depretere et al. 3 the linear and rotational pulses were recalculated as equivalent sinusoidal pulses. In this study as in the study of Cui et al. 1 the complete linear and rotational raw pulses from each of the 8 cases were used as inputs. 


\section{Results}

The resulting comparison between the original (Oka model) and the mean angles model (Musigazi model) is shown in table 2 when the rupture criterion is the mean ultimate strain, the mean plus one standard deviation or minus one standard deviation.. The success rate is the percentage of the correct predictions to the total number of experiments. The unsuccessful predictions are further separated into false positive (FP) or false negative (FN) rupture predictions, with false negative being less desirable from a head protection point of view.

Table 2: Rupture prediction results of the cadaver impact simulations

\begin{tabular}{c|ccc|ccc|rcc}
\hline \hline & \multicolumn{3}{|c|}{ Success rate } & \multicolumn{3}{c|}{ False negatives } & \multicolumn{3}{c}{ False positives } \\
& $\mu-\sigma$ & $\mu$ & $\mu+\sigma$ & $\mu-\sigma$ & $\mu$ & $\mu+\sigma$ & $\mu-\sigma$ & $\mu$ & $\mu+\sigma$ \\
\hline Oka model & $75 \%$ & $87.5 \%$ & $87.5 \%$ & 1 & 1 & 1 & 1 & 0 & 0 \\
Musigazi model & $75 \%$ & $75 \%$ & $87.5 \%$ & 1 & 1 & 1 & 1 & 1 & 0 \\
Max Angles model & $75 \%$ & $75 \%$ & $75 \%$ & 1 & 1 & 2 & 1 & 1 & 0 \\
Min Angles model & $75 \%$ & $75 \%$ & $87.5 \%$ & 1 & 1 & 1 & 1 & 1 & 0 \\
\hline
\end{tabular}

In table 3 the individual results for each case are shown for all models using the mean ultimate strain as rupture criterion. For the same cases the same rupture prediction comes as an outcome from all models except for the case of 01-3_1. The mean angles model and the minimum angles model give a false positive whereas for the original (Oka model) and the maximum angles model there is a successful prediction if we consider the ultimate strain as a binary criterion. There instead of a false positive there is a successful prediction for the original(Olia model) and the Maximum angles model, which makes the success rate $87.5 \%$ for these two-models, eren throughthe maximum strain seen is 3 times higher in the original model.

The location of the maximum BV strains in the Musigazi model (mean angles) is mainly at the middle cortical segments $(4,5,6,7)$ (see figure 1), which correspond to the rupture sites reported by Depreitere et al. 3 . Instead, the Oka model (original) yields the maximum BV strains almost completely at the back of the cortical segments $(8,10)(8,9)$.

Table 3: Detailed results of the cadaver impact simulations

\begin{tabular}{|c|c|c|c|c|c|c|c|c|c|c|c|c|}
\hline \multirow{2}{*}{$\begin{array}{l}\text { Specimen } \\
\text { Num. }\end{array}$} & \multicolumn{3}{|c|}{ Oka model } & \multicolumn{3}{|c|}{ Musigazi model } & \multicolumn{3}{|c|}{ Max Angles Musigazi } & \multicolumn{3}{|c|}{ Min Angles Musigazi } \\
\hline & $\mathrm{RP}$ & M. Str & Loc & $R P$ & M. Str & Loc & $\mathrm{RP}$ & M. Str & Loc & $\mathrm{RP}$ & M. Str & Loc \\
\hline 01-3_1 & $\mathrm{SP}$ & 25.78 & $\mathrm{M}$ & FP & 35.19 & $\mathrm{M}$ & SP & 8.08 & $\mathrm{M}$ & $\mathrm{FP}$ & 35.49 & $\mathrm{M}$ \\
\hline 21-3_1 & $\mathrm{SP}$ & 5.00 & B & SP & 4.26 & B & SP & 1.19 & B & SP & 7.13 & M \\
\hline 22-3_1 & $\mathrm{SP}$ & 5.62 & B & $\mathrm{SP}$ & 4.07 & M & SP & 1.13 & B & SP & 6.61 & M \\
\hline 25-2_1 & $\mathrm{SP}$ & 6.61 & B & $\mathrm{SP}$ & 5.83 & F & $\mathrm{SP}$ & 1.74 & F & $\mathrm{SP}$ & 7.00 & M \\
\hline 28-2_1 & $\mathrm{FN}$ & 9.94 & B & $\mathrm{FN}$ & 9.67 & M & FN & 2.44 & B & $\mathrm{FN}$ & 15.12 & M \\
\hline 29-3_1 & $\mathrm{SP}$ & 46.20 & B & SP & 48.69 & M & SP & 32.19 & B & $\mathrm{SP}$ & 51.24 & M \\
\hline 30-2_1 & $\mathrm{SP}$ & 7.68 & B & SP & 5.17 & M & SP & 1.94 & B & $\mathrm{SP}$ & 9.63 & M \\
\hline 32-2_1 & $\mathrm{SP}$ & 8.49 & B & SP & 5.40 & M & $\mathrm{SP}$ & 2.07 & B & $\mathrm{SP}$ & 9.90 & M \\
\hline
\end{tabular}

The minimum observed angles model shows consistently higher BV maximum strains that range 
from $4 \%$ to $44 \%$ with maximum strains located at the middle cortical segments i.e. $(4,5,6,7)$. The maximum observed angles model shows consistently lower BV maximum strains that range from $151 \%$ to $439 \%$ and the location of the maximum strain being more at the back segments i.e. $(8,10)$ $(9,10)$.

As it can be seen by both tables 3 and 4 , the resulting maximum strain rupture location sites show that the models with the mean entry angles and minimum entry configurations locate the maximum strains in the middle segments which is where all ruptures happened in the cadaver experiments.

Table 4: Rupture location prediction results of the cadaver impact simulations

\begin{tabular}{cccc}
\hline \hline Model & Frontal M. Str Rupture & Middle M. Str Rupture & Back M. Str Rupture \\
${$\cline { 1 - 3 }$} }$ & $0 \%$ & $12.5 \%$ & $87.5 \%$ \\
Musigazi model & $12.5 \%$ & $75 \%$ & $12.5 \%$ \\
Max Ang. Musigazi & $12.5 \%$ & $12.5 \%$ & $75 \%$ \\
Min Ang. Musigazi & $0 \%$ & $100 \%$ & $0 \%$ \\
\hline
\end{tabular}

\section{Discussion}

In this study, updated information on the location and entry angles obtained from a much greater statistical sample were used to model BVs in the KTH head model Then a comparison was made by simulating the cadaver experiments performed by Depreitere et al. 2 both using the original model that used the Oka dataset 10 and the updated models that used the Musigazi dataset 9 .

The dataset of Musigazi contains in vivo measurements of a group of 78 patients which is much larger compared to the 10 cadavers examined by Oka. This higher statistical power is probably the reason why, in the dataset of Musigazi, there are not so extreme angles especially for the veins located at the frontal and occipital parts of the brain, as it can also be seen in Fig. 2.

This could also explain why the rupture location prediction is better when the dataset of Musigazi is used. The extreme angles that have been reported by Oka for the occipital regions and have been implemented in the original KTH model make this group of BV elements more vulnerable to impacts on the back of the head.

Even though there is no improvement in successful predictions, the improvement in maximum strain location is a step forward. The fact that the repositioning improves the location prediction of the ruptures suggests that the Musigazi model, by offering more plausible layout of BVs than the Oka model outputs results with a higher likelihood. is more biofidelic than the Olka model.

Unfortunately, there are no imaging data available that show the subject specific BV configuration in the cadaver experiments. The full body cadaver experiments are also from a relatively small statistical sample and it is likely that they are not representing the full range of the population. Thus, even if the beam elements are placed in the mean position and angle, taking into account the considerable inter-specimen variation means that the model with the Musigazi mean dataset could be representing the population better than the experimental data do.

In addition, the models with the extreme angles show that there can be significant differences on the strain applied on the BVs depending on their entry angle. The maximum strain location in the maximum angles model is located at the back segments which can be explained by the fact 
that the entry angles in the occipital region of this model are significantly smaller than the veins in the rest of the models.

When simulating the 01-3_1 case of the cadaver experiments, if we dont consider a range of one standard deviation for the ultimate strain, then it seems that the Oka model (original) has a higher predictive capability than the mean angles model. For the 01-3_1 case, it seems that the Oka model (original) is behaving better. That can be debated. The same cadaver was impacted a second time, since no rupture was detected the first time. That was case 01-3_2. This second case was simulated both with the original and the mean angles model and did not gave a rupture in both models. The peak rotational acceleration of 01-3_1 was $15156 \mathrm{rad}^{2} / \mathrm{s}$ with a duration of $12 \mathrm{~ms}$ when the 01-3_2 had $9708 \mathrm{rad}^{2} / \mathrm{s}$ with a duration of $12.6 \mathrm{~ms}$, i.e. in the first impact the head experienced much higher accelerations yet, in the experiments, it was on the second impact that there was a rupture. That could mean that there was significant damage sustained when the cadaver was impacted the first time the was not captured when examined in between the two impacts i.e. between 01-3_1 and 01-3_2. The 01-3_2 case was excluded from results because it was considered untrustworthy.

Furthermore, there are still a number of factors that need to be considered. According to the Musigazi dataset more than $80 \%$ of the BVs are located in the middle segments. This could mean that even with 78 patients the actual number of veins located to the back and front segments were not enough to give statistically strong mean values. Moreover, Musigazi used CT angiograms where it is possible that some of the smaller BVs were not detected whereas in the study of Oka it is almost certain that all the existing BVs have been detected due to the fact that it was an autopsy. All of the ruptures occurred on cadavers with an age of higher than 80 years old. Eren if the beam elements are placed in the mean pesition and angle, there is a considerable

inter-specimen variation both in number and position. Unfortunately there are no imaging data available that could-show the subject specific BV configuration in the eadarer experiments.

The BV geometry is oversimplified and does not show any shearing deformation especially at the SSS connection point. Furthermore, BVs are known to contain a high percentage of collagen which makes the material behaviour to be nonlinear and anisotropic. The linear material definition used in this model does not capture this behaviour which again affects considerably the way the vein deforms in a combined tension and shear deformation.

The BV material model used is linear elastic, which does not show the anisotrepic nature of the tissue, and also the vein geometry is oversimplified. Finally, the mean ultimate strain was used as a failure criterion eren though the reported-standard-deviations-of ultimate-strain are alse-signifieantly large.

In order to improve further the behaviour of the head model, a more complex, in vivo, geometry could show the complex loading conditions these veins experience under impact. A material model that can capture the anisotropic behaviour of this soft tissue could also improve the overall behaviour. Other improvements at the head models that could also affect ASDH predictability could be detailed geometry brain models that include sulci and gyri. Having a surface that interacts with the CSF which is not smooth will surely affect the CSF displacements having a direct impact on the BV strain. To investigate this a detailed geometry brain model that includes sulci and gyri and a brain skull interface with a fluid structure interaction is needed. Developing a BV rupture risk assessment by taking into account the whole ultimate strain distribution and assigning a risk factor can also be a way to deal with the uncertainty involving the ultimate strain rupture criterion. ean also be a way forward.

Finally, more experimental or accident reconstruction data are needed to verify the level of accuracy in both rupture prediction and rupture location prediction. 


\section{Conclusion}

The 4 different models had similar predictive capability. The location of the maximum strain was closer to the rupture sites reported in the experiments, for the models with the Mean angles and the Minimum angles of the Musigazi dataset.

In order to predict ASDH due to BV rupture a higher level of biofidelity in BV representation is still needed.The materials and geometries used until now are far too simplified to capture the true behaviour of BVs under impact conditions.

\section{Conflict of interest statement}

The authors have no conflicts of interest arising from the direct applications of this research.

\section{Acknowledgements}

This work was supported by the European Unions Horizon 2020 research and innovation programme under the Marie Skodowska-Curie grant agreement No. 642662 for HEADS ITN. 


\section{References}

[1] Cui, Z. Y., Famaey, N., Depreitere, B., Ivens, J., Kleiven, S., Vander Sloten, J., 2017. On the assessment of bridging vein rupture associated acute subdural hematoma through finite element analysis. Computer Methods in Biomechanics and Biomedical Engineering 5842.

[2] Depreitere, B., Van Lierde, C., Maene, S., Plets, C., Sloten, J. V., Van Audekercke, R., Van der Perre, G., Goffin, J., 2004. Bicycle-related head injury: A study of 86 cases. Accident Analysis and Prevention 36 (4), 561-567.

[3] Depreitere, B., Van Lierde, C., Vander Sloten, J., Van Audekercke, R., Van der Perre, G., Plets, C., Goffin, J., 2006. Mechanics of acute subdural hematomas resulting from bridging vein rupture. Neurosurgery 104, 950-956.

[4] Georgeta, A., Baeck, K., Verbeken, E., Verpoest, I., Vander, J., Goffin, J., Depreitere, B., 2014. The biomechanical behaviour of the bridging vein superior sagittal sinus complex with implications for the mechanopathology of acute subdural haematoma. Journal of the Mechanical Behavior of Biomedical Materials 32, 155-165. URL http://dx.doi.org/10.1016/j.jmbbm.2013.12.007

[5] Horgan, T. J., Gilchrist, M. D., 2003. The creation of three-dimensional finite element models for simulating head impact biomechanics. Int J Crashworthiness 8 (4), 353-366.

[6] Kleiven, S., 2007. Predictors for traumatic brain injuries evaluated through accident reconstructions. Stapp Car Crash (September).

[7] Ls-dyna, R., 2015. USER' S MANUAL. Vol. I.

[8] Maxeiner, H., 1997. Detection of ruptured cerebral bridging veins at autopsy. Forensic Science International 89, 103-110.

[9] Musigazi, G. U., Cui, Z. Y., Vander Sloten, J., Depreitere, B., 2013. Combining 2d and 3dcomputed tomography to explore bridging vein entrance into the superior sagittal sinusimproving acute subdural hematoma prevention.

[10] Oka, K., Rhoton, A. L., Barry, M., Rodriguez, R., 1985. Microsurgical Anatomy of the Superficial Veins of the Cerebrum. Forensic Science International 17 (5).

[11] Takhounts, E. G., Ridella, S. A., Tannous, R. E., Campbell, J. Q., Malone, D., Danelson, K., Stitzel, J., Rowson, S., Duma, S., 2003. Investigation of Traumatic Brain Injuries Using the Next Generation of Simulated Injury Monitor ( SIMon ) Finite Element Head Model. Int J

[12] Viano, D. C., Casson, I. R., Pellman, E. J., Zhang, L., King, A. I., Yang, K. H., 2005. Concussion in professional football: Brain responses by finite element analysis: Part 9. Neurosurgery 57 (5), 891-916.

URL http://dx .doi.org/10.1227/01 . NEU.0000186950.54075.3B 